\title{
Effect of commercially purified deoxynivalenol and zearalenone mycotoxins on microbial diversity of pig cecum contents
}

\author{
Kondreddy Eswar Reddy ${ }^{1}$, Minji Kim ${ }^{1}$, Ki Hyun Kim¹, Sang Yun $\mathrm{Ji}^{1}$, Youlchang Baek ${ }^{1}$, Ju Lan Chun ${ }^{1}$, \\ Hyun Jung Jung ${ }^{1,2}$, Changyong Choe ${ }^{3}$, Hyun Jeong Lee ${ }^{1,4}$, Minseok Kim ${ }^{1,5, *}$, and Sung Dae Lee ${ }^{1, *}$
}

\footnotetext{
* Corresponding Authors:

Minseok Kim

Tel: +82-62-530-2128, Fax: +82-62-530-2129

E-mail: mkim2276@jnu.ac.kr

Sung Dae Lee

Tel: +82-63-238-7454, Fax: +82-63-238-7497

E-mail: leesd@korea.kr

${ }^{1}$ Animal Nutrition and Physiology Team, National Institute of Animal Science, Rural Development Administration, Wanju 55365, Korea

2 Swine Science Division, National Institute of Animal Science, Rural Development Administration, Cheonan 31000, Korea ${ }^{3}$ Division of Animal Disease and Health, National Institute of Animal Science, Rural Development Administration, Wanju 55365, Korea

${ }^{4}$ Dairy Science Division, National Institute of Animal Science, Rural Development Administration, Cheonan 31000, Korea

${ }^{5}$ Department of Animal Science, College of Agriculture and Life Science, Chonnam National University, Gwangju 61186, Korea

\section{ORCID}

Kondreddy Eswar Reddy

https://orcid.org/0000-0003-2024-7724 Minji Kim

https://orcid.org/0000-0003-2106-1921

Ki Hyun Kim

https://orcid.org/0000-0002-9834-2126

Sang Yun Ji

https://orcid.org/0000-0001-7235-3655

Youlchang Baek

https://orcid.org/0000-0003-4454-5339

Ju Lan Chun

https://orcid.org/0000-0002-4618-586X

Hyun Jung Jung

https://orcid.org/0000-0002-7004-2017

Changyong Choe

https://orcid.org/0000-0003-4222-3360

Hyun Jeong Lee

https://orcid.org/0000-0002-2312-9048

Minseok Kim

https://orcid.org/0000-0002-8802-5661

Sung Dae Lee

https://orcid.org/0000-0002-9167-4099
}

Submitted Mar 3, 2020; Revised Mar 30, 2020; Accepted Apr 27, 2020
Objective: Deoxynivalenol (DON) and zearalenone (ZEN) are mycotoxins that frequently contaminate maize and grain cereals, imposing risks to the health of both humans and animals and leading to economic losses. The gut microbiome has been shown to help combat the effects of such toxins, with certain microorganisms reported to contribute significantly to the detoxification process.

Methods: We examined the cecum contents of three different dietary groups of pigs (control, as well as diets contaminated with $8 \mathrm{mg}$ DON $/ \mathrm{kg}$ feed or $0.8 \mathrm{mg} \mathrm{ZEN} / \mathrm{kg}$ feed). Bacterial $16 \mathrm{~S}$ rRNA gene amplicons were acquired from the cecum contents and evaluated by nextgeneration sequencing.

Results: A total of 2,539,288 sequences were generated with $\sim 500$ nucleotide read lengths. Firmicutes, Bacteroidetes, and Proteobacteria were the dominant phyla, occupying more than $96 \%$ of all three groups. Lactobacillus, Bacteroides, Megasphaera, and Campylobacter showed potential as biomarkers for each group. Particularly, Lactobacillus and Bacteroides were more abundant in the DON and ZEN groups than in the control. Additionally, 52,414 operational taxonomic units were detected in the three groups; those of Bacteroides, Lactobacillus, Campylobacter, and Prevotella were most dominant and significantly varied between groups. Hence, contamination of feed by DON and ZEN affected the cecum microbiota, while Lactobacillus and Bacteroides were highly abundant and positively influenced the host physiology.

Conclusion: Lactobacillus and Bacteroides play key roles in the process of detoxification and improving the immune response. We, therefore, believe that these results may be useful for determining whether disturbances in the intestinal microflora, such as the toxic effects of DON and ZEN, can be treated by modulating the intestinal bacterial flora.

Keywords: Mycotoxins; Pig; Intestine; Deoxynivalenol; Zearalenone; Detoxification

\section{INTRODUCTION}

Deoxynivalenol (DON) and zearalenone (ZEN) are mycotoxins produced by Fusarium fungi, which frequently contaminate maize and grain cereals [1]. These mycotoxins have been widely investigated due to their universal distribution and capacity to cause pathological changes in humans and farm animals. Mycotoxicosis is the term used to refer to diseases caused by these toxins in humans and animals, often presenting in farm animals as reduced feed intake, poor feed conversion, feed refusal, reduced body weight gain, immune suppression, poor reproductive capacity, and long-term chronic effects, ultimately resulting in economic losses.

Numerous mechanisms have been suggested to explain the biological effects elicited by DON-contaminated feed. For instance, long-term exposure to DON may cause anorexia, reduced feed intake and weight gain, decreased nutritional efficiency, and immune mod- 
ulation [2,3]. According to Pestka and Smolinski [4], DON inhibits protein biosynthesis and induces pro-inflammatory cytokine production. Among farm animals, pigs are the most sensitive to DON with chronic exposure, characterized as 1 to $2 \mathrm{mg} \mathrm{DON} / \mathrm{kg}$ in feed, resulting in decreased appetite; whereas $3 \mathrm{mg}$ DON/kg reduces body temperature and induces variations in the gastric wall of piglets. Further, according to Reddy et al [3], consumption of a diet containing $8 \mathrm{mg} \mathrm{DON} / \mathrm{kg}$ for four weeks decreased body weight gain, reduced feed conversion rate, affected inflammatory cytokine production, reduced immunoglobulin (Ig)G, IgM, and serotonin values, and decreased total antioxidant contents in serum samples of pigs, as well as histopathological damage in kidney and liver samples compared to a standard dietary group. Furthermore, the usual growth rate of pigs has been reported to be reduced by approximately $7 \%$ for each $\mathrm{mg}$ of $\mathrm{DON} / \mathrm{kg}$ increase in the diet, which may vary depending on numerous factors. Meanwhile, complete refusal of feed occurs at concentrations greater than $12 \mathrm{mg} \mathrm{DON} / \mathrm{kg}$. According to Young et al [5], high doses of DON (0.1 to $0.3 \mathrm{mg} / \mathrm{kg}$ body weight or $20 \mathrm{mg} / \mathrm{kg}$ of feed) in piglets led to vomiting. When pigs consume DON it becomes absorbed in the proximal aspect of the small intestine; and once in the colon de-epoxidation can occur, however, detoxification is not significantly induced [6].

The ZEN is a phytoestrogenic compound with estrogenic effects in farm animals [7]. The ZEN is associated with various dose-dependent mycotoxicoses in farm animals, particularly pigs. Pigs generally show clinical signs at low doses of ZEN (1.5 to $2 \mathrm{mg} / \mathrm{kg}$ in diet) including vaginal and vulvar swelling and thickness, increased uterus mass, testicular atrophy, and expansion of the mammary glands, as well as other reproductive effects, such as decreased fertility, increased number of resorptions, and reduced litter size [7]. Typically, hyperestrogenism and mortality are observed in pigs fed high doses of ZEN over an extended period of time [8], and infertility was observed at concentrations higher than $100 \mathrm{mg}$ ZEN/kg [9]. A recent study [3] showed that in pigs fed a $0.8 \mathrm{mg} Z \mathrm{ZEN} / \mathrm{kg}$ diet for four weeks, the serum IgG and IgM levels decreased; meanwhile total antioxidant levels decreased in the serum yet increased in urine. At the same ZEN dose, inflammatory cytokine and chemokine marker expression was reduced in the kidney and liver tissues, with microscopic lesions appearing in these tissues.

The gastrointestinal (GI) microbiota plays essential roles in animals, with a strong association reported between the host and its GI microbiota, particularly in immune response, energy uptake from food, activity of important metabolites, resistance to toxins, and metabolic products of fermentation. However, few studies have examined the GI microbiota in farm animals [10], particularly the effects of DON and ZEN mycotoxins on the pig intestinal microbiota communities. Different species colonize various areas of the GI tract to dif- ferent degrees. Pigs, specifically, are highly sensitive to dietary mycotoxins, which target the mucus and microbiota [11]. For instance, Fusarium toxins can cause gut tissue damage, shorten the height of villi, and cause dysbiosis of gut microbiota [12]. We previously showed that Fusarium mycotoxins primarily influence the composition of pig gut microbiota, ultimately causing activation of intestinal inflammation [10].

Among the various gut microbiota, bacteria play a major role in the GI tract. Next-generation DNA sequencing (NGS) approaches are effective for studying the composition of the host microbiota, with the high read abundance of amplified sequences providing insight into microbial diversity. Advanced molecular biology techniques can be useful in examining complex microbiological communities, such as those in the gut that cannot be cultured outside of the host. Moreover, these approaches have revealed variations in the gut bacterial community between functional sites, individuals, as well as healthy and diseased conditions [13]. Importantly, studies of the gut microbiota have also demonstrated its critical role in maintaining animal and human health.

The direct effects exerted by consumption of highly concentrated and commercially-purified DON and ZEN on the composition of the cecum microbiota have not been reported in pigs. Therefore, this study was performed to determine the effect of commercially-purified Fusarium mycotoxins, DON and ZEN, on individual pigs after four weeks of feeding, as well as to compare mycotoxin-treated pig groups to gain insight into the quantitative and qualitative composition of the pig cecum microbiota. These results may be useful for determining whether disturbances in the intestinal microflora, such as the toxic effects of DON and ZEN, can be ameliorated via modulating the intestinal bacterial flora.

\section{MATERIALS AND METHODS}

\section{Ethics statement}

The protocols for the animal experimental procedures were reviewed and accepted by the Institutional Animal Care and Use Committee of the National Institute of Animal Science, South Korea (No. 2015-147).

\section{Experimental design and animal exposure to DON and ZEN}

This study was conducted using 14 male castrated 8-weekold piglets (Landrace $\times$ Yorkshire $=$ Large White Landrace; $\sim 19 \mathrm{~kg}$ ), obtained from a commercial pig farm. Each pig was housed in an individual pen $(2.1 \times 1.4 \mathrm{~m})$ at $25^{\circ} \mathrm{C} \pm 1^{\circ} \mathrm{C}$. The pigs were allowed to adjust to their new housing conditions for 1 week, and then assigned to three dietary groups, control $(\mathrm{n}=4), \mathrm{DON}(\mathrm{n}=5)$, and ZEN $(\mathrm{n}=5)$ groups, with approximately equal body weights. The control group was 
fed a standard diet to meet the nutritional requirements for piglets (Table 1) [14], whereas the two treatment groups were fed a standard diet plus DON ( $8 \mathrm{mg} / \mathrm{kg})$ or ZEN $(0.8 \mathrm{mg} / \mathrm{kg})$ for 4 weeks. The commercial DON and ZEN powders were added as purified toxins (Biomin Pte. Ltd., Singapore) and properly mixed into the diet at 8 and $0.8 \mathrm{mg} / \mathrm{kg}$, respectively. The control feeds, DON-and ZEN-purified mycotoxins contaminated feeds, and water were provided ad libitum throughout the 4-week experimental period.

\section{Mycotoxins analysis from the feed}

The DON and ZEN in the supplemented corn feeds were quantitatively evaluated by ultra-performance liquid chromatography (UPLC). Briefly, a homogenized DON- or ZENmixed grain sample $(1 \mathrm{~g})$ was extracted with $20 \mathrm{~mL}$ of distilled water and shaken for $30 \mathrm{~min}$ (DON samples) or with $0.5 \mathrm{~g}$ of $\mathrm{NaCl}$ and $20 \mathrm{~mL}$ acetonitrile and shaken for $1 \mathrm{~h}$ (ZEN sample). After filtering the extract through Whatman paper (No. 1), $5 \mathrm{~mL}$ of the DON filtrate sample was diluted in $20 \mathrm{~mL}$ of phosphate-buffered saline, and $5 \mathrm{~mL}$ of the ZEN filtrate sample

Table 1. Ingredients and chemical composition of pig standard diet (as-fed basis)

\begin{tabular}{lc}
\hline Item & Control diet \\
\hline Ingredients (\%) & \\
Ground corn & 58.56 \\
Soybean meal (46\% crude protein) & 14.00 \\
Extruded soybean meal & 12.00 \\
Whey powder (12\% crude protein) & 7.00 \\
Fish meal & 3.45 \\
Soybean oil & 1.60 \\
L-lysine·HCl (78\%) & 0.43 \\
DL-methionine (99\%) & 0.14 \\
L-threonine (99\%) & 0.12 \\
Calcium hydrophosphate & 1.08 \\
Limestone & 0.60 \\
Choline chloride (50\%) & 0.20 \\
Sodium chloride & 0.32 \\
Vitamin-trace mineral premix & 0.50 \\
Calculated nutrients (\%) & \\
Metabolizable energy (kcal/kg) & 3,444 \\
Crude fiber & 2.29 \\
Crude protein & 20.78 \\
Crude fat & 3.44 \\
Ash & 4.35 \\
Lysine & 1.47 \\
Methionine & 0.49 \\
Calcium & 0.75 \\
Phosphorus & 0.45 \\
\hline Provided the folowing quanties per $\mathrm{kg}$ of complete & \\
\hline
\end{tabular}

1) Provided the following quantities per $\mathrm{kg}$ of complete diet: vitamin, $\mathrm{A}$ 11,000 IU; vitamin $\mathrm{D}_{3}, 1,500 \mathrm{IU}$; vitamin $\mathrm{E}, 44.1 \mathrm{IU}$; vitamin $\mathrm{K}_{3}, 4.0 \mathrm{mg}$; vitamin $B_{1}, 1.4$ mg; vitamin $B_{2}, 5.22$ mg; vitamin $B_{5}, 20.0$ mg; vitamin $B_{12}, 0.01$ mg; niacin, $26.0 \mathrm{mg}$; pantothenic acid, $14 \mathrm{mg}$; folic acid, $0.8 \mathrm{mg}$; biotin, $44 \mathrm{mg} ; \mathrm{Fe}, 100.0 \mathrm{mg}$ as iron sulfate; $\mathrm{Cu}, 16.50 \mathrm{mg}$ as copper sulfate; $\mathrm{Zn}$, $90.0 \mathrm{mg}$ as zinc sulfate; $\mathrm{Mn}, 35.0 \mathrm{mg}$ as manganese sulfate; I, $0.30 \mathrm{mg}$ as calcium iodate. was diluted in $20 \mathrm{~mL}$ of $1 \%$ Tween 20 solution. The extracted DON and ZEN samples were loaded separately onto immunoaffinity chromatography columns. UPLC methods and mass spectrometry were performed as described previously by Reddy et al [15]. The limit of detection (LOD) was $5 \mu \mathrm{g} / \mathrm{kg}$ for both DON and ZEN, and the limit of quantification was $10 \mu \mathrm{g} / \mathrm{kg}$ for DON and $16.7 \mu \mathrm{g} / \mathrm{kg}$ for ZEN. Analysis of the standard diet as well as the DON and ZEN-contaminated feed was repeated three times. We found that the total amount of DON and ZEN in the mixed corn feed was $7.38 \mathrm{mg} / \mathrm{kg}$ and $0.67 \mathrm{mg} / \mathrm{kg}$ feed, respectively, which are similar to that of the original concentrations of mixed corn feed. Alternatively, the control diet was free of mycotoxins or it may have simply contained levels below the LOD.

\section{Sampling and processing}

A total of 14 castrated male piglets were used to study the cecum microbiota composition in the standard diet and DON and ZEN mycotoxin-contaminated feed groups. After 4 weeks, standard diet pigs and pigs fed a diet containing DON and ZEN were sacrificed by anesthetic pentobarbital overdose. Following complete cardiac arrest, the cecum contents of all pigs were aseptically collected directly into sample containers, quickly frozen in liquid nitrogen, and stored at $-80^{\circ} \mathrm{C}$ until analysis.

\section{DNA extraction and 16S rRNA gene sequencing}

DNA was extracted from the pig cecum contents of each group by the repeated bead beating plus column method [16], and stored at $-20^{\circ} \mathrm{C}$. From each DNA sample, 14 amplicon libraries were produced using the 341F (5'-CCTACGGGNGGCWG CAG-3') and 805R (5'-GACTACHVGGGTATCTAATCC $-3^{\prime}$ ) primers, which typically produce approximately 450 -base pair products. The resulting 14 amplicon libraries were constructed using a paired-end protocol with the MiSeq platform (Illumina, San Diego, CA, USA) at the Macrogen sequencing facility (Macrogen, Inc., Seoul, Korea). Paired reads were collected using the FLASH program [17]. The assembled sequences were demultiplexed using the default parameters and quality filtered using a Q20 minimum value with the QIIME software package 1.9.1 [18]. According to DeSantis et al [19], the taxa were determined using the Greengenes reference database, while operational taxonomic units (OTUs) were determined at $97 \%$ sequence similarity using the uclust program [20]. After 100,000 sequences were subsampled from each cecum sample to normalize the number of OTUs, alpha diversity indices, including the number of OTUs, Chaol, PD_whole_tree distance, and Shannon diversity index were determined.

\section{Statistical analysis}

Taxa (or OTUs) accounting for an average of $\leq 0.2 \%$ of the 
total sequences were considered as major taxa (or OTUs) and statistically analyzed. The taxonomic composition of each taxon or OTU in the total sequences was compared among the control, DON, and ZEN dietary groups by analysis of variance, followed by Duncan's test using XLSTAT statistical software version 18.07 (Addinsoft, New York, NY, USA). A significant difference was considered at $\mathrm{p}<0.05$.

\section{RESULTS}

\section{Sequencing and bacterial abundance}

Quality control of all cleaned 16S rRNA sequencing ( $r r s$ ) reads among the pig cecum contents in the control, DON, and ZEN dietary treatment groups resulted in identification of 2,539,288 sequences with $~ 500$-nucleotide read lengths. From these group sequences, 714,639 represented control dietary group cecum contents, whereas 890,094 and 934,555 represented DON and ZEN dietary treated cecum contents, respectively. The numbers of $r$ rs sequences from individual control dietary cecum samples were 171,037 to 187,601; those from the DON-dietary treated cecum samples were 155,260 to 189,289 , and those from ZEN-dietary treated samples were 146,729 to 212,732 .

We used principal coordinate analysis (PCoA; Figure 1) with weighted uniFrac distances to compare the sample structures. Most DON samples showed an anterior distribution and maintained a certain distance from the posterior control and ZEN dietary samples. Two ZEN-treated samples

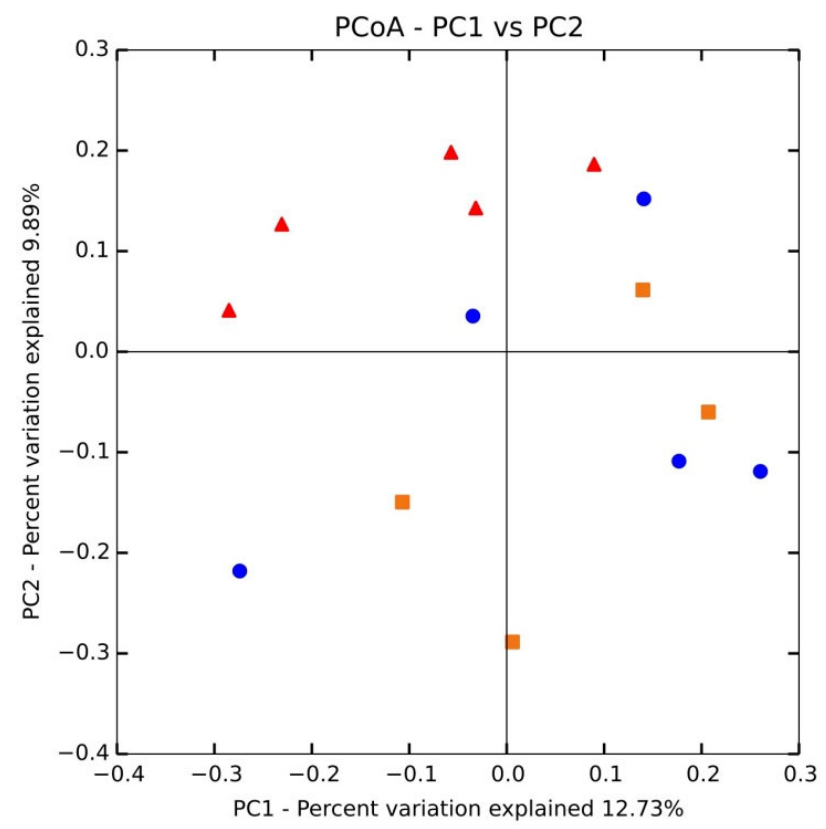

Figure 1. Principal coordinates analysis ( $P C O A)$ of cecum microbial communities after different dietary treatments. PCoA based on the weighted UniFrac distance of 16s rRNA samples of cecum bacteria in the control (orange dots), DON (red dots), and ZEN (blue dots) dietary treatment groups. DON, deoxynivalenol; ZEN, zearalenone. were nearer to DON and clearly separated from the control group. These results suggest intra-group variation.

\section{Bacterial taxonomic composition}

All rrs sequences from the cecum contents of the control, DON, and ZEN dietary groups differed at the phylum, family, and genus levels (Figure 2A, 2C, Supplementary Table S1). The microbial data for all three dietary groups were examined to determine the mean relative abundance (taxon reads/total reads in a sample). The phyla Firmicutes and Bacteroidetes were the most abundant in the three groups, accounting for $56.8 \%$ and $35.6 \%$ of all $r r s$ sequences, respectively (Figure 2A). The third richest phylum was Proteobacteria, accounting for $4.3 \%$ of the total sequences in the three groups. The remaining identified phyla showed $<1.0 \%$ abundance of all $r r s$ sequences and included Spirochetes $(0.9 \%)$ Cyanobacteria $(0.9 \%)$, Actinobacteria $(0.8 \%)$, TM7 $(0.4 \%)$, Tenericutes $(0.3 \%)$, Verrucomicrobia $(0.2 \%)$, and Fusobacteria $(0.1 \%)$ (Supplementary Table S1). Significant differences were found only in the phylum Verrucomicrobia among the dietary treatment groups.

As shown in Table 2, at the family level, Prevotellaceae was highly abundant, accounting for $21.4 \%$ of the collective data; the remaining most abundant families were Ruminococcaceae (14.7\%), Lachnospiraceae (13.6\%), Veillonellaceae (11.1\%), Bacteroidaceae (4.1\%), Lactobacillaceae (4.0\%), Erysipelotrichaceae (3.8\%), and Clostridiaceae (2.0\%). The other 21 families detected represented $<2.0 \%$ of all dietary group sequences (Table 2). Among the families, Bacteroidaceae (4.1\%), Lactobacillaceae (4.0\%), Clostridiaceae (2.0\%), Campylobacteraceae $(1.4 \%)$, Aeromonadaceae $(0.5 \%)$, Chlamydomonadaceae $(0.4 \%)$, Pasteurellaceae $(0.3 \%)$, Turicibacteraceae $(0.2 \%)$, Rhodocyclaceae $(0.2 \%)$, Verrucomicrobiaceae $(0.1 \%)$, Christensenellaceae $(0.1 \%)$, and Flavobacteriaceae $(0.1 \%)$ showed significant differences $(\mathrm{p}<0.05)$ among the dietary treatment groups. Most families with significant differences were more abundant in the DON and ZEN treatment groups than in the control. Particularly, Lactobacillaceae showed a high abundance in the DON dietary group (6.5\%) and ZEN dietary group (3.8\%) compared to the control group (1.1\%). Bacteroidaceae also showed a high abundance in both the DON and ZEN dietary groups (4.6\% and 5.3\%, respectively) compared to the control (2.0\%). However, Clostridiaceae showed high abundance in the control (3.0\%) and ZEN (2.7\%) dietary groups compared to in the DON group (0.4\%). Similarly, Campylobacteraceae was abundant in the control (1.5\%) and ZEN (2.8\%) groups, whereas this family was not detected in the DON dietary group. The families Clostridiaceae, Pasteurellaceae, and Turicibacteraceae families showed high abundance in the ZEN group and very low abundance in the DON group compared to in the control group. 
A

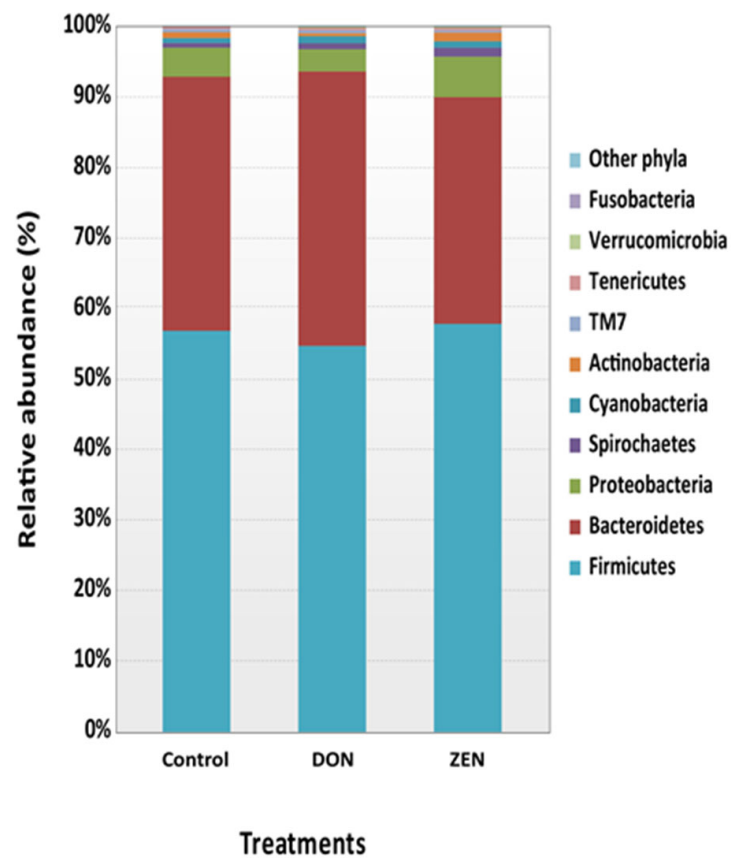

C

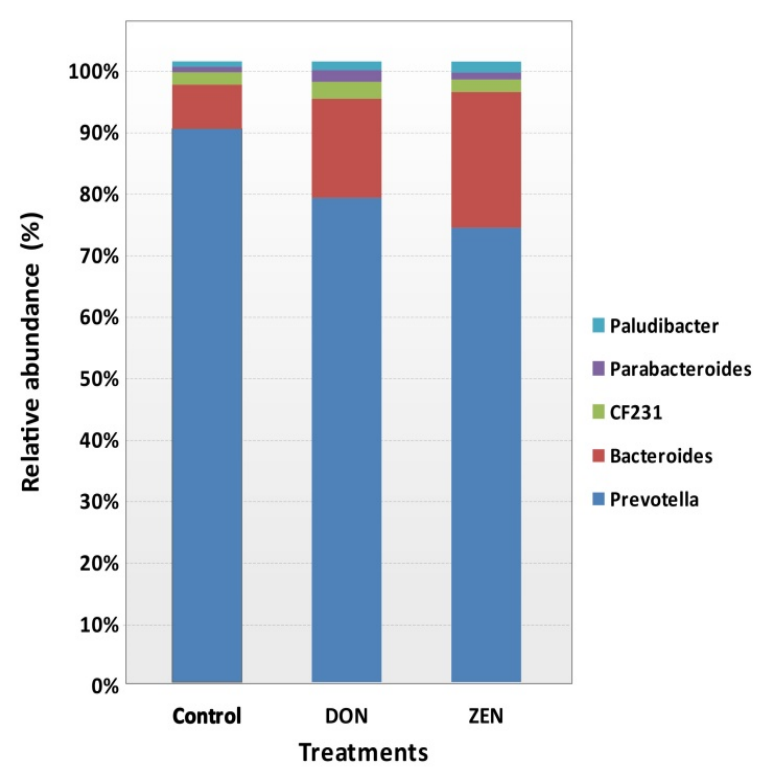

B

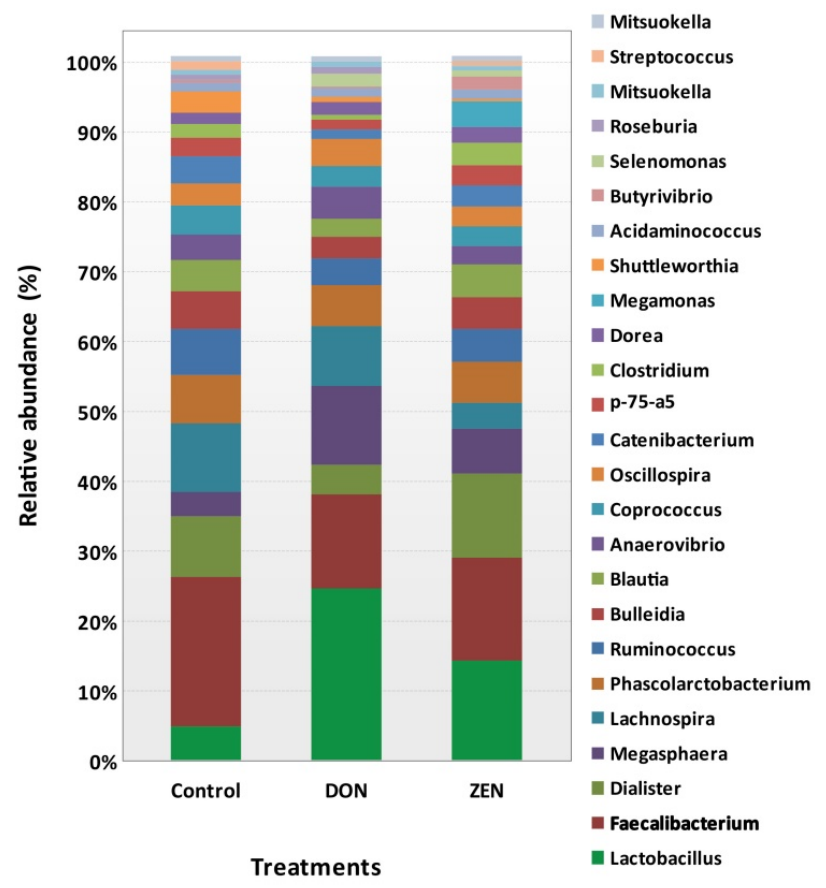

Figure 2. Relative abundances of microbial taxa in the cecum at the phylum and genus levels in the control, deoxynivalenol (DON), and zearalenone (ZEN) dietary treatment groups. (A) Relative abundances of taxa within all phyla. (B) Abundance of Firmicutes. (C) Abundance of Bacteroidetes.

Variations in the abundance of the ten phyla between the dietary treatments are shown in Figure 3A, and the top 15 genera with the highest richness variation between the control, DON, and ZEN dietary treatment groups are shown in Figure 3B. Prevotella was highly abundant at the genus level in the three dietary groups accounting for $21.4 \%$ of the 2,539,288 rrs sequences. Other genera, represented by at least $0.5 \%$ of the total three dietary group sequences, were Faecalibacterium (4.4\%) Bacteroides (4.1\%), Lactobacillus (4.0\%), Dialister (2.3\%), Megasphaera (2.0\%) Lachnospira (1.9\%) Phascolarc- tobacterium (1.7\%), Campylobacter (1.4\%), Ruminococcus (1.3\%), Bulleidia (1.2\%), Blautia (1.1\%), Anaerovibrio (0.9\%), Coprococcus (0.9\%), Oscillospira (0.9\%), Treponema (0.9\%), Catenibacterium (0.7\%), p-75-a5 (0.6\%), CF231 (0.6\%), and Clostridium (0.5\%). Differences in the abundance of phyla among the dietary groups were observed; however, few of these differences were significant between the control and treatment groups (Supplementary Table S1).

The phylum Firmicutes was most abundant and collectively occupied $56.8 \%$ of phyla in the three dietary groups, 
Table 2. Relative microbial abundance of cecum major family taxa among control, deoxynivalenol and zearalenone dietary treatment groups

\begin{tabular}{|c|c|c|c|c|c|c|}
\hline \multirow{2}{*}{ Family name } & \multicolumn{4}{|c|}{ Percentage of total sequences ${ }^{1)}$} & \multirow{2}{*}{ SEM } & \multirow{2}{*}{ p-value } \\
\hline & Collective data $^{2)}$ & Control & DON & ZEN & & \\
\hline Prevotellaceae & 21.4 & 25.4 & 22.2 & 17.5 & 0.088 & 0.395 \\
\hline Ruminococcaceae & 14.7 & 15.9 & 13.4 & 14.9 & 0.030 & 0.276 \\
\hline Lachnospiraceae & 13.6 & 14.4 & 14.1 & 12.3 & 0.034 & 0.309 \\
\hline Veillonellaceae & 11.1 & 9.6 & 10.6 & 12.8 & 0.068 & 0.623 \\
\hline Bacteroidaceae & 4.1 & $2.0^{b}$ & $4.6^{\mathrm{a}}$ & $5.3^{\mathrm{a}}$ & 0.064 & 0.001 \\
\hline Lactobacillaceae & 4.0 & $1.1^{\mathrm{b}}$ & $6.5^{\mathrm{a}}$ & $3.8^{\mathrm{a}}$ & 0.138 & 0.005 \\
\hline Erysipelotrichaceae & 3.8 & 4.9 & 2.0 & 4.7 & 0.105 & 0.098 \\
\hline Clostridiaceae & 2.0 & $3.0^{\mathrm{a}}$ & $0.4^{\mathrm{b}}$ & $2.7^{\mathrm{a}}$ & 0.109 & 0.001 \\
\hline S24-7 & 1.9 & 1.6 & 2.5 & 1.5 & 0.120 & 0.538 \\
\hline Campylobacteraceae & 1.4 & $1.5^{\mathrm{a}}$ & $0.0^{\mathrm{b}}$ & $2.8^{\mathrm{a}}$ & 0.422 & 0.019 \\
\hline Spirochaetaceae & 0.9 & 0.7 & 0.8 & 1.3 & 0.362 & 0.796 \\
\hline Coriobacteriaceae & 0.8 & 0.8 & 0.4 & 1.2 & 0.135 & 0.186 \\
\hline Porphyromonadaceae & 0.8 & 0.5 & 1.0 & 0.8 & 0.109 & 0.115 \\
\hline Succinivibrionaceae & 0.7 & 0.7 & 0.6 & 0.7 & 0.211 & 0.887 \\
\hline Aeromonadaceae & 0.5 & $0.2^{b}$ & $0.6^{\mathrm{a}}$ & $0.6^{\mathrm{a}}$ & 0.062 & 0.001 \\
\hline Chlamydomonadaceae & 0.4 & $0.2^{b}$ & $0.5^{\mathrm{a}}$ & $0.5^{\mathrm{a}}$ & 0.069 & 0.001 \\
\hline F16 & 0.4 & 0.4 & 0.4 & 0.3 & 0.295 & 0.542 \\
\hline Desulfovibrionaceae & 0.3 & 0.3 & 0.3 & 0.3 & 0.130 & 0.681 \\
\hline Enterobacteriaceae & 0.3 & 0.5 & 0.2 & 0.2 & 0.141 & 0.996 \\
\hline Pasteurellaceae & 0.3 & $0.4^{\mathrm{a}}$ & $0.0^{b}$ & $0.4^{\mathrm{a}}$ & 0.394 & 0.010 \\
\hline Streptococcaceae & 0.2 & 0.3 & 0.0 & 0.2 & 0.260 & 0.448 \\
\hline Turicibacteraceae & 0.2 & $0.3^{\mathrm{a}}$ & $0.0^{\mathrm{b}}$ & $0.2^{\mathrm{a}}$ & 0.291 & 0.001 \\
\hline Rhodocyclaceae & 0.2 & $0.1^{\mathrm{b}}$ & $0.2^{\mathrm{a}}$ & $0.2^{\mathrm{a}}$ & 0.173 & 0.001 \\
\hline
\end{tabular}

with values of $56.7 \%, 54.07 \%$, and $57.8 \%$ in the control, DON, and ZEN groups, respectively (Supplementary Table S1). Twenty-six genera within Firmicutes were detected, which comprised at least $0.2 \%$ of all sequences in each dietary group; mainly, Faecalibacterium (4.4\%) and Lactobacillus (4.0\%) showed high abundance compared to the other genera. Faecalibacterium was detected at $5.5 \%, 3.6 \%$, and $4.2 \%$ in the control, DON, and ZEN dietary groups, respectively, with no significant difference between groups. The collective data of Lactobacillus abundance was $4.0 \%$, with significant differences $(p=0.005)$ observed between the dietary treatments. Interestingly, Lactobacillus levels were nearly six-fold higher in the DON (6.5\%) group, and more than three-fold higher in the ZEN (3.8\%) group compared to in the control (1.1\%). Megasphaera also showed significantly high abundance $(\mathrm{p}=$ 0.05 ) and comprised $2.0 \%$ of all sequences, with higher abundance in the DON (3.0\%) and ZEN (1.8\%) treatment groups than in the control (0.9\%). Peptococcus and Pelosinus also showed significant differences $(\mathrm{p}<0.05)$ among the dietary treatments. Peptococcus showed $0.2 \%$ abundance in the control and $0.1 \%$ in ZEN, with no abundance in DON (0.0\%); whereas Pelosinus showed $0.2 \%$ abundance in the DON group, $0.1 \%$ in the ZEN group, and $0.0 \%$ in the control group. The major Firmicutes species identified were Faecalibacterium prausnitzii (4.4\%), Ruminococcus bromii (0.2\%), and Selenomonas ruminantium $(0.2 \%)$, which did not differ $(\mathrm{p}>0.05)$ in abundance between the three dietary groups.

The phylum Bacteroidetes was second most abundant and collectively occupied $35.6 \%$ of the total sequences. Bacteroidetes showed $35.9 \%, 38.8 \%$, and $32.0 \%$ in the control, DON and ZEN dietary treatments, respectively. In Bacteroidetes, the most abundant genera were Prevotella, Bacteroides, CF231, Parabacteroides, and Paludibacter. Prevotella showed high abundance with $25.4 \%$ and $22.2 \%$ in the control and DON groups, with a low abundance of $17.5 \%$ in the ZEN group ( $>0.05)$. Bacteroides showed high abundance (4.1\%) and significant differences $(\mathrm{p}=0.005)$ among the dietary groups, at $2.0 \%$ in the control group; the DON (4.6\%) and ZEN (5.3\%) dietary groups showed more than two-fold higher levels compared to the control group. Paludibacter also showed significant differences between dietary groups, with both the DON and ZEN dietary groups exhibiting twofold higher abundance $(0.4 \%)$ compared to the control group (0.2\%). The major Bacteroidetes species identified were Prevotella copri (10.7\%) and Prevotella stercorea (1.5\%), however, their abundance did not differ $(\mathrm{p}>0.05)$ among 
A

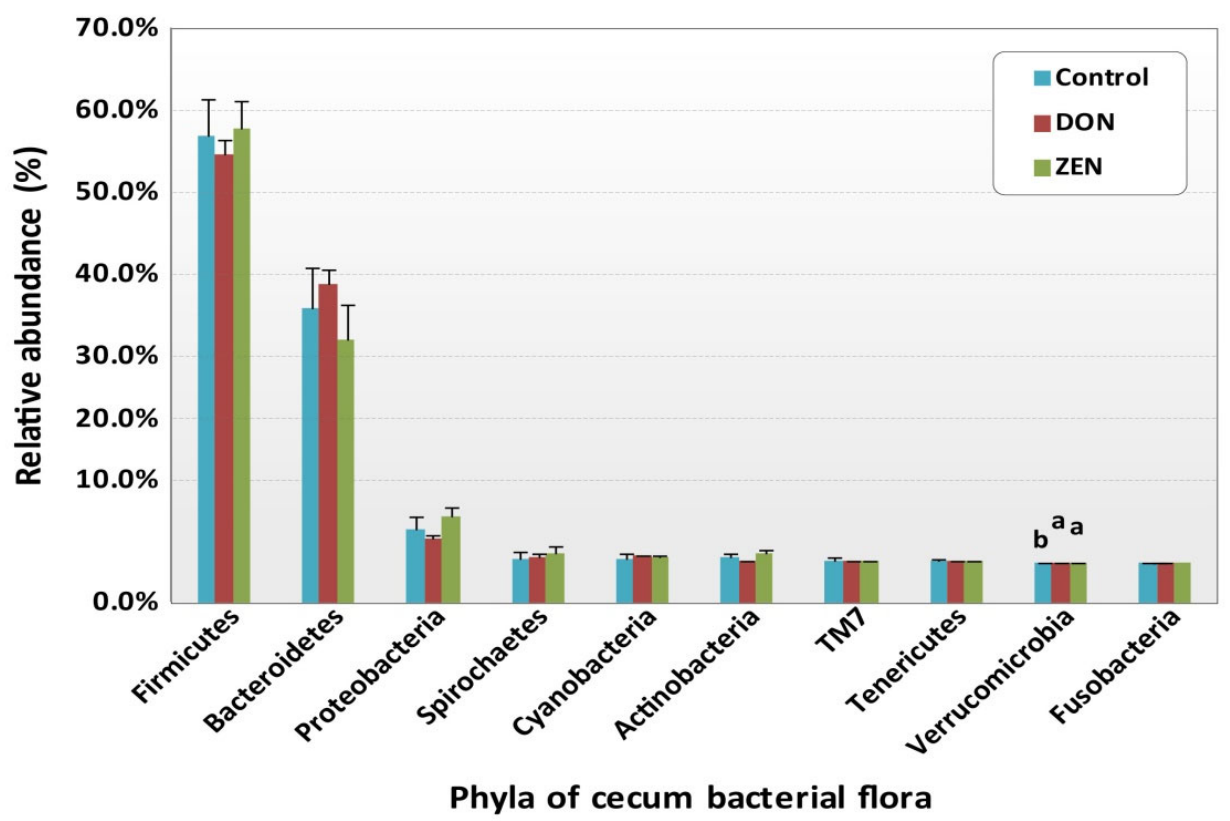

B

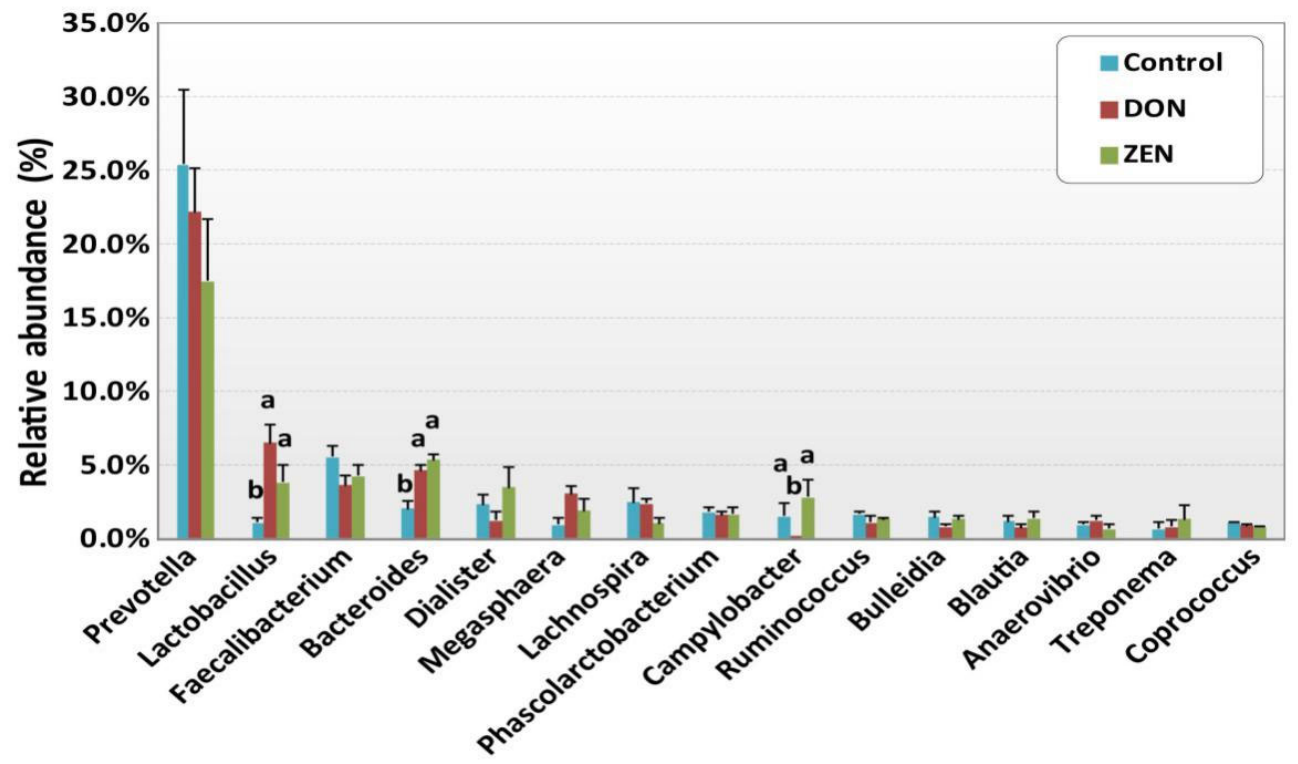

Genera of cecum bacterial flora

Figure 3. Relative abundances of the cecum microbiota among the control and deoxynivalenol (DON) and zearalenone (ZEN) mycotoxin groups (A) Variations in the relative abundance of the cecum microbiota at the phylum level. (B) Differences in the relative abundance of the top 15 cecum bacteria at the genus level. Different letters indicate significant differences $(p<0.05)$.

the three dietary groups.

Proteobacteria was the third most prevalent phylum (4.3\%). Within this phylum, we found five genera: Campylobacter, Succinivibrio, Actinobacillus, Desulfovibrio, and Dechloromonas, of which Campylobacter showed significantly high abundance in the ZEN (2.8\%) and control groups (1.5\%), with no abundance in the DON group (0.0\%). Actinobacillus and Dechloromonas also showed significant differences among the dietary groups as did Turicibacter, in the phylum
Actinobacteria, and Akkermansia, in the phylum Verrucomicrobia.

Analysis of operational taxonomic unit diversity A total of 52,415 OTUs were found among the three dietary treatment group samples at a 0.03 dissimilarity cutoff level. All sequence reads were normalized and examined using the Shannon diversity index, Chaol richness estimator, and Simpson index. The normalized samples did not vary in 
richness ( $p>0.05$ ) for the numbers of OTUs among the three dietary treatment groups, however, significant differences were observed $(\mathrm{p}=0.05)$ between the double sequence reads (Table 3). Seventeen of the 52,415 OTUs were significantly $(p<0.05)$ represented by $\geq 0.2 \%$ of all sequences in at least one dietary treatment group, whereas seven of the 17 were classified into known genera, however, were not classified to any known species. The remaining ten OTUs could not be classified into known genera (Table 4). The major OTUs accounting $\geq 0.2 \%$ of all sequences were classified to species Prevotella copri, Prevotella copri, Prevotella stercorea, Faecalibacterium prausnitzii, or Ruminococcus bromii, however, their prevalence did not differ $(\mathrm{p}<0.05)$ among the three dietary treatment groups.

Among the 17 OTUs, Bacteroides (denovo163744) was the most dominant genera in the collective data, showing the highest prevalence in the DON and ZEN dietary groups and lowest in the control group. Similarly, another OTU also belonging to Bacteroides (denovo64865) was abundant in the DON and ZEN dietary groups, with low abundance in the control. Lactobacillus was the second most abundant OTU (denovo41137) and was much higher in the DON group, and moderately higher in the ZEN, compared to the control dietary group (Table 4). The next most abundant OTU was that representing the genus Campylobacter (denovo8890), which showed the greatest richness in the ZEN and control and lowest in the DON dietary groups. One OTU, classified as Prevotella (denovo47686), showed approximately five-fold higher abundance in ZEN and two-fold higher in DON compared to the control dietary group. The genus Paludibacter OTU (denovo77418) was also abundant in the DON and ZEN dietary groups compared to in the control group. The abundance of two unclassified Clostridiaceae OTU families (denovo3971 and denovo47258), and OTU Bulleidia genera

Table 3. Diversity statistics of standard diet (control), deoxynivalenol, and zearalenone dietary treatment groups

\begin{tabular}{|c|c|c|c|c|c|c|c|}
\hline Sample group & $\begin{array}{c}\text { No. of observed } \\
\text { OTUs }{ }^{1)}\end{array}$ & Singles & Doubles & Chao1 & $\begin{array}{l}\text { Phylogenetic } \\
\text { diversity whole tree }\end{array}$ & Shannon & Simpson group \\
\hline Control $(n=4)$ & $12,618.50^{\mathrm{a}}$ & $8,970.00^{\mathrm{a}}$ & $1,458.25^{b}$ & $40,210.65^{a}$ & $565.86^{a}$ & $8.97^{a}$ & $0.98^{\mathrm{a}}$ \\
\hline $\operatorname{DON}(\mathrm{n}=5)$ & $14,162.60^{a}$ & $10,125.80^{a}$ & $1,662.40^{\mathrm{a}}$ & $45,046.49^{a}$ & $626.61^{a}$ & $9.26^{\mathrm{a}}$ & $0.98^{\mathrm{a}}$ \\
\hline ZEN $(\mathrm{n}=5)$ & $13,519.40^{a}$ & $9,582.80^{a}$ & $1,599.80^{a b}$ & $42,238.81^{a}$ & $600.85^{a}$ & $9.11^{\mathrm{a}}$ & $0.98^{\mathrm{a}}$ \\
\hline $\mathrm{Pr}>\mathrm{F}$ & 0.15 & 0.20 & 0.05 & 0.35 & 0.17 & 0.35 & 0.96 \\
\hline
\end{tabular}

OTUs, operational taxonomic units; DON, deoxynivalenol; ZEN, zearalenone.

1) Means among the three dietary groups were compared by analysis of variance, followed by Duncan's test.

${ }^{a b}$ Values with different superscript letters in the same column are significantly different $(p<0.05)$.

Table 4. Relative microbial abundances of significantly different operational taxonomic units, calculated for the control, deoxynivalenol, and zearalenone dietary treatment groups

\begin{tabular}{|c|c|c|c|c|c|c|c|}
\hline \multirow{2}{*}{ OTU ID ${ }^{1)}$} & \multirow{2}{*}{ Classification } & \multicolumn{4}{|c|}{ Percentage of total sequences ${ }^{2)}$} & \multirow{2}{*}{ SEM } & \multirow{2}{*}{ p-value } \\
\hline & & Collective data & Control & DON & ZEN & & \\
\hline denovo163744 & Bacteroides & 2.84 & $1.37^{\mathrm{b}}$ & $3.12^{\mathrm{a}}$ & $3.74^{\mathrm{a}}$ & 0.069 & 0.002 \\
\hline denovo8890 & Campylobacter & 1.04 & $1.24^{\mathrm{a}}$ & $0.01^{b}$ & $1.92^{\mathrm{a}}$ & 0.466 & 0.013 \\
\hline denovo164640 & Prevotella & 0.91 & $1.79^{\mathrm{a}}$ & $0.00^{\mathrm{b}}$ & $1.12^{\mathrm{a}}$ & 0.593 & 0.008 \\
\hline denovo3971 & Unclassified Clostridiaceae & 0.46 & $1.00^{\mathrm{a}}$ & $0.06^{b}$ & $0.43^{\mathrm{a}}$ & 0.222 & 0.002 \\
\hline denovo147371 & Unclassified Erysipelotrichacea & 0.39 & $0.67^{\mathrm{a}}$ & $0.16^{\mathrm{ab}}$ & $0.41^{b}$ & 0.119 & 0.023 \\
\hline denovo129399 & Unclassified Lachnospiraceae & 0.33 & $0.16^{b}$ & $0.43^{\mathrm{a}}$ & $0.37^{\mathrm{a}}$ & 0.062 & 0.002 \\
\hline denovo153523 & Unclassified Ruminococcaceae & 0.29 & $0.53^{\mathrm{a}}$ & $0.31^{\mathrm{a}}$ & $0.10^{b}$ & 0.146 & 0.015 \\
\hline denovo6037 & Unclassified Chlamydomonadaceae & 0.29 & $0.13^{b}$ & $0.36^{\mathrm{a}}$ & $0.35^{\mathrm{a}}$ & 0.070 & 0.001 \\
\hline denovo24662 & Unclassified Lachnospiraceae & 0.23 & $0.25^{\mathrm{ab}}$ & $0.34^{\mathrm{a}}$ & $0.10^{\mathrm{b}}$ & 0.144 & 0.041 \\
\hline denovo77418 & Paludibacter & 0.18 & $0.09^{b}$ & $0.22^{\mathrm{a}}$ & $0.21^{\mathrm{a}}$ & 0.071 & 0.003 \\
\hline
\end{tabular}

OTUs, operational taxonomic units; DON, deoxynivalenol; ZEN, zearalenone; SEM, standard error of the mean.

${ }^{1)}$ A total of 52,414 de novo OTUs were identified in the three dietary groups in consecutive order.

2) Values indicates the means.

${ }^{a b}$ Values with different superscript letters in the same row are significantly different $(p<0.05)$. 
(denovo116445) were highly prevalent in the control and ZEN dietary groups and lowest in the DON dietary treatment group. Three unclassified OTU families of Lachnospiraceae (denovo129399), Chlamydomonadaceae (denovo6037), and Aeromonadaceae (denovo48653) were much more abundant in both the DON and ZEN treatment groups compared to the control. Two other unclassified Lachnospiraceae OTU families (denovo24662 and denovo92328) showed significantly higher levels in the DON dietary treatment than in the ZEN and control groups. The abundance of unclassified OTUs Clostridiales (denovo14126), Erysipelotrichacea (denovo147371), and Ruminococcaceae (denovo153523), was much lower in the DON and ZEN treatment groups compared to the control.

\section{DISCUSSION}

We assessed the effect of diets contaminated with DON and ZEN on the cecum microflora of pigs and evaluated which bacterial strains may reduce the toxicological effects of DON and ZEN. The GI microbiota in pigs has not been completely defined as this dynamic community contains many hundreds of species, comprised primarily of anaerobic bacteria [21]. The DON and ZEN mycotoxins have been shown to negatively affect GI microbiota, affected the GI species composition and bacterial numbers in pigs to varying degrees depending on the mycotoxin concentrations in diet, treatment period, animal age, location of toxins in the GI, and nutritional dietary factors. Although previous studies have examined the effects of feed naturally-contaminated with mycotoxins on the gut microbiota of pigs [22,23], here we sought to analyze the cecum contents of pigs fed commercially-purified DON and ZEN mycotoxins to compare microbial taxonomic abundances in the control, DON, and ZEN dietary treatment groups.

Among the phyla detected, Firmicutes (56.8\%) and Bacteroidetes $(35.6 \%)$ were highly abundant (Figure 3A), occupying more than $92 \%$ of the cecum contents from the DON, ZEN, and control dietary groups, however, their abundances showed no significant differences between dietary groups (Figure 2A, Supplementary Table S1). Similarly, in our previous study, the colons of DON and ZEN dietary-treated pigs also showed greater than $90 \%$ Firmicutes and Bacteroidetes $(p>0.05)$ [10]. Typically, the compositions of the intestinal microbiota in the cecum and colon are similar among pigs [24]. However, $\mathrm{Li}$ et al [23] found that naturally DON-contaminated wheat fed to pigs caused differences in the abundance of Firmicutes and Bacteroidetes in the cecum, colon, and ileum. Further, Isaacson and Kim [24], evaluated naturally weaned pigs and found that Firmicutes and Bacteroidetes accounted for more than $90 \%$ of the bacteria detected in the cecum contents. Meanwhile, in another study, Firmicutes and Bacteroidetes comprised more than $90 \%$ of bacteria in pigs fed a fuminosincontaminated diet (12 mg/kg feed) [25]. In the current study, no significant differences were noted at the phylum level in the toxic dietary treatment groups. However, if the dietary treatment period were to be extended, the microbiota may be altered in the toxin treatment groups. Family-level cecum bacterial abundances are shown in Table 2, and significant families in these genera were identified.

Among the cecum contents, specific genera were identified as potentially significant biomarkers for differentiating between the control and the DON and ZEN mycotoxin dietary treatment groups. Specifically, Lactobacillus, Bacteroides, and Megasphaera were significantly more abundant in the cecum microbiota of the DON and ZEN dietary groups compared to the control group (Figure 3b). Lactobacillus accounted for a predominant genus in DON and was moderately abundant in ZEN, meanwhile its levels were very low in the control group (Figure 2A). Similar results were observed in our previous study regarding the pattern of Lactobacillus abundance in the colons of pigs [10], with differences observed between the cecum and colon contents in the DON, ZEN, and control dietary treatments. It has been suggested that a myriad of factors contribute to the microbial shifts occurring between the cecum and colon, including stress resulting from mycotoxin-contaminated feed, chemical composition of the diet, as well as various physiological factors [26]. Recently, the abundance of Lactobacillus was found to be increased by $13 \%$ in the cecum of pigs fed DON- contaminated wheat in combination with Clostridium sp. WJ06 [23]. In another study, $15.8 \%$ of Lactobacillus sequences were recovered from the pig intestinal samples, indicating their important roles in the gut on host physiology [27]. Similarly, through 16 S rRNA analysis, Niu et al [28] demonstrated that Lactobacillus is one of the most prevalent genera in pig intestinal samples, irrespective of age. Furthermore, NGS technology revealed Lactobacillus as a key member of the fecal microbiota in all growth stages of pigs.

Lactobacillus species are considered to be probiotics that play a key role in various physiological functions of their hosts, including microbial interference, antimicrobial properties, supplementary influences on nutrition, antitumor effects, decreasing cholesterol in the host serum, and immunomodulatory influences [29]. Particularly, in pigs, a positive effect was observed on Lactobacillus abundance following feed limitation. According to Yang et al [30], Lactobacillus can support the development of an optimized microbiome by enhancing the richness and number of lactobacilli and other native probiotic bacteria. The primary indigenous probiotic bacteria can promote growth and immunity of piglets through positive cascade signal transduction pathways. The piglet body provides a tolerant habitat and nutrients for bacterial colonization and growth, in return, probiotics generally 
generate prebiotics such as short-chain fatty acids and bacteriocins that can improve growth, and decrease the risk of enteric diseases caused by pathogens or toxins, while also enhancing the host feed utilization capacity. Furthermore, Walter [31] indicated that autochthonous Lactobacillus are often used as probiotics due to their natural capacity to survive harsh physiological conditions, including the acidic stomach, pancreatic enzymes, and bile salts during their passage through the GI tract $[32,33]$. These Lactobacillus probiotic bacteria were also selected for their capacity to adhere to mucus and epithelial cells, as these characteristics are required for their effective colonization of the gut's mucosal and epithelial layer and to increase their competitiveness against pathogens [34]. This competitive profile is likely conferred by autochthonous Lactobacillus, which is used as a biomarker of health in the pig gut microflora.

According to many studies, the large quantity of lactic acid bacteria (LAB) in the digestive tract is associated with the age of pigs and feeding of probiotics. LAB play a crucial role in the host, generating substances such as acetic, butyric, and propionic acid, as well as other similar short-chain fatty acids, B vitamins, and amino acids, including bacteriocins ad antimicrobial metabolites. Colonization of the pig digestive tract by $\mathrm{LAB}$ is prevented by various pathogens stimulating the immune system of the host [35]. Moreover, LAB can bind Fusarium mycotoxins in their environment [36]. According to Franco et al [37], the capacity of viable and heat-inactivated Lactobacillus cells was reduced by more than $60 \%$ in the presence of $1.5 \mu \mathrm{g} / \mathrm{mL} \mathrm{DON}$ in liquid media. However, according to Yang et al [38], Lactobacillus plays a key role in removing ZEN in vitro; meanwhile El-Nezami et al [39] reported the binding affinity of ZEN, and its derivative a-ZEN, with two food-grade strains of Lactobacillus. Still further, although the mechanisms of aflatoxin binding by specific Lactobacillus are unclear, cell wall peptidoglycans and polysaccharides have been suggested as the two most significant elements responsible for binding by Lactobacillus and the absorption of mutagens or carcinogens in the intestine [40]. In this study, we predicted that Lactobacillus concentrations were higher in DON and ZEN dietary treatment groups and that LAB may contribute significantly to the detoxification of DON and ZEN in the pig intestine. However, the intestinal mucus and its resident microbiota are important targets of dietary mycotoxins, particularly DON [11]. Consequently the GI mucus can reduce the ability of probiotics, such as Lactobacillus, to bind mycotoxins, thereby interfering with their adsorption of dietary mycotoxin. Similarly, in the current study, regular administration of the probiotic Lactobacillus during the dietary treatment period may have reduced the effect of mucus. The number of bacterial colonyforming units may have reduced the effects of mucus on the adsorption of DON and ZEN dietary mycotoxins by the
Lactobacillus cell wall.

In the current study, Bacteroides accounted for another predominate genus, which was more abundant in the DON and ZEN dietary toxin groups than in the control. Bacteroides spp. are considered as a source of novel useful bacteria for treating gut immune dysfunctions, colitis, and metabolic disorders, as well as for cancer prevention [41]. Bacteroides spp. are dominant and play a key role in pig intestine, making them a major group in pig feces. Similar to our results, Saint-Cyr et al [42] demonstrated that feeding with DON at $100 \mathrm{mg} / \mathrm{kg}$ body weight for 4 weeks by oral gavage increased Bacteroides levels in rat intestines. Other studies in pigs supported the use of prebiotics for selecting Bacteroides spp. This capacity to influence the microbiota also involves inclusion of a specific level of fermentable fiber in the pig diet, which stimulates colonic fermentation, as well as the presence of exogenous enzymes, which create oligosaccharides with prebiotic effects from non-starch polysaccharides [43]. Moreover, Bacteroides spp. also help to protect against gut colonization by various potential pathogens. Based on these results, Bacteroides may play a key role in eradicating DON and ZEN from the pig gut. In this study, Megasphaera was approximately three-fold more abundant in the DON group, and two-fold higher in the ZEN group, compared to the control. Conversely in our previous study we reported that Megasphaera was more abundant in the ZEN dietary group, with no significant differences observed between the ZEN and control groups [10]. Megasphaera spp. are abundant and important in the human gut microbiota, and their capacity to produce important metabolites indicates beneficial health effects on the host. Megasphaera elsdenii was reported to comprise approximately $0.12 \%$ to $5.9 \%$ of bacteria in pig feces [44], where it uses both L- and ${ }_{\mathrm{D}}$-lactate and increases short-chain fatty acids, which are crucial for pig colonocyte development and proliferation, as well as small intestine growth [45]. We, therefore, hypothesized that Megasphaera abundance increases in the intestines of pigs in response to the lactate produced by abundant Lactobacillus, and may positively influence intestinal disorders or immune responses following DON and ZEN dietary treatment of pigs. However, few studies have examined the effects of dietary mycotoxins on these bacteria in animals.

Other bacterial genera, including Campylobacter, Paludibacter, Turibacter, Peptococcus, Pelosinus, Actinobacillus, Dechloromonas, and Akkermansia, also showed significant differences between the dietary treatment groups. Campylobacter is the most common cause of GI infection in pigs, characterized by inflammation and diarrhea involving cramps, fever, and pain [46]. According to Burrough et al [47], Paludibacter is abundant in the pig intestine and proficiently ferments complex polysaccharides produced by the pig. We found that DON and ZEN influence the composition 
and fermentation products of the pig intestinal microbiota, thus affecting the health and performance of the pig. However, further studies are needed to determine the functions of these genera.

In the current study, 17 OTUs were identified that differed significantly in abundance between the control, DON, and ZEN dietary treatment groups. Similarly, our previous study of pig colon contents also showed varying OTUs abundances between these groups [10]. However, in the current study, most OTUs in the cecum differed from those in the colon. Specifically, Bacteroides, Lactobacillus, and Campylobacter OTUs were more abundant in the cecum than in the colon content; we also identified three unclassified Lachnospiraceae, and two unclassified Clostridiaceae family OTUs which differed in abundance between the DON and ZEN dietary groups. According to Przybylska-Gornowicz et al [12], the response of the intestinal immune system is unambiguous in the cecum; however, variable and sometimes difficult to interpret results were obtained in the ascending colon and descending colon. Unclassified Lachnospiraceae showed higher abundance in both DON and ZEN dietary treatment groups. Moreover, similar to our results, Gratz et al [48] found that the abundance of Lachnospiraceae was higher in the cecum contents of DON dietary pigs; no previous studies evaluated ZEN dietary pig treatment groups. Lachnospiraceae also showed higher abundance in fecal samples from pigs fed fumonisin in the diet [25]. Lachnospiraceae bacteria may also play a key role in healthy pigs and serve to improve the pig immune system. Compared to the control, unclassified Clostridiaceae was less abundant in DON, however, did not exhibit differences in abundance with the ZEN dietary group. Compared to that reported previously for the colon contents, here we demonstrate that DON OTU abundance was decreased in the cecum, while ZEN did not differ from the control group. According to Piotrowska et al [22], Clostridium abundance is reduced in the colons of both the DON and ZEN dietary groups in gilts, suggesting that the abundance of Clostridiaceae bacteria was inhibited by the highly toxic DON, with no significant effects imposed by ZEN, which has lower toxicity. Prevotella OTU was also significantly decreased in the DON group, without significant differences observed in the ZEN group compared to the control. Conversely, in our previous study we reported significantly higher OTU abundances in both the DON and ZEN dietary groups [15], with a higher Prevotella abundance in pigs fed fumonisin $(12 \mathrm{mg} / \mathrm{kg}$ feed). The Prevotella-driven enterotype appears to be important in subjects who consumed high levels of carbohydrates and fiber. In this study, due to the high concentrations of DON and ZED, the daily feed intake was reduced in the DON group compared to in the ZEN and control groups, which subsequently reduced the abundance of Prevotella in the
DON due to the reduced consumption of carbohydrates.

Since mycotoxins can alter the microbial composition balance of the pig intestine, a complete understanding of the relationship between pigs and their gut microbiota will facilitate the development of new dietary treatments that can increase pig growth, protect piglets from pathogenic bacteria, and enhance host feed utilization. In the current study, specific bacteria genera were highly abundant in both the DON and ZEN dietary treatments compared to in the control; in this case, this microbiota may have positively impacted the host physiology. Other bacteria in the DON and ZEN dietary treatments, particularly in the DON group, showed a lower abundance compared to the control; in these cases, DON and ZEN may have induced lesions in the cecum by disturbing the integrity of the pig intestinal barrier and reducing the abundance of specific microbiota. These results show that the composition and structure of the pig cecum greatly differs compared to that determined in our previous study of microflora in the pig colon. Similarly, a previous study also strongly demonstrated differences between cecum and colon microbial data in pigs [23]. DON and ZEN may alter susceptibility to infectious diseases in humans and animals by affecting gut health as well as the innate and adaptive immune systems. However, the mechanisms by which mycotoxins affect the intestinal microbiota composition remain unclear.

In conclusion, the results of this study indicate that the GI bacterial flora in pigs became disrupted following consumption of feed contaminated with commercial DON and ZEN for four weeks. The genera Lactobacillus (particularly in DON) and Bacteroides dominated the bacterial flora in both the DON and ZEN dietary treatments. In addition, OTUs assigned to unclassified Lachnospiraceae belonging to Firmicutes, were more abundant in both the DON and ZEN dietary treatments than in the control group. Based on the present data, there may be potential opportunities to isolate and characterize useful probiotics that decrease the level of mycotoxins and help restore intestinal microbiota that have been disturbed by mycotoxins.

\section{CONFLICT OF INTEREST}

We certify that there is no conflict of interest with any financial organization regarding the material discussed in the manuscript.

\section{ACKNOWLEDGMENTS}

This work was carried out with the support of the Cooperative Research Program for Agriculture Science \& Technology Development (Project No. PJ01093202) and the 2017 RDA Fellowship Program of the National Institute of Animal Sciences, Rural Development Administration, Republic of Korea. 


\section{REFERENCES}

1. Marin S, Ramos AJ, Cano-Sancho G, Sanchis, V. Mycotoxins: occurrence, toxicology, and exposure assessment. Food Chem Toxicol 2013;60:218-37. https://doi.org/10.1016/j.fct.2013. 07.047

2. Pinton P, Oswald IP. Effect of deoxynivalenol and other type B trichothecenes on the intestine: a review. Toxins 2014;6: 1615-43. https://doi.org/10.3390/toxins6051615

3. Reddy KE, Song J, Lee HJ, et al. Effects of high levels of deoxynivalenol and zearalenone on growth performance, and hematological and immunological parameters in pigs. Toxins 2018;10:114. https://doi.org/10.3390/toxins10030114

4. Pestka JJ, Smolinski AT. Deoxynivalenol: toxicology and potential effects on humans. J Toxicol Environ Health B 2005; 8:39-69. https://doi.org/10.1080/10937400590889458

5. Young LG, McGirr L, Valli VE, Lumsden JH, Lun A. Vomitoxin in corn fed to young pigs. J Anim Sci 1983;57:655-64. https://doi.org/10.2527/jas1983.573655x

6. Dänicke S, Valenta H, Döll S. On the toxicokinetics and the metabolism of deoxynivalenol (don) in the pig. Arch Anim Nutr 2004;58:169-80. https://doi.org/10.1080/00039420410 001667548

7. Nesic K, Stevanovic J, Sinovec Z. Efficacy of mineral and organic adsorbent in alleviating harmful effects of zearalenone on pig blood serum protein status. Vet Glas 2008;62:25-34. https://doi.org/10.2298/VETGL0802025N

8. Zinedine A, Soriano JM, Molto JC, Manes J. Review on the toxicity, occurrence, metabolism, detoxification, regulations and intake of zearalenone: an oestrogenic mycotoxin. Food Chem Toxicol 2007;45:1-18. https://doi.org/10.1016/j.fct.2006. 07.030

9. Tiemann U, Danicke S. In vivo and in vitro effects of the mycotoxins zearalenone and deoxynivalenol on different non-reproductive and reproductive organs in female pigs: a review. Food Addit Contam 2007;24:306-14. https://doi.org/ 10.1080/02652030601053626

10. Reddy KE, Jeong JY, Song J, et al. Colon microbiome of pigs fed diet contaminated with commercial purified deoxynivalenol and zearalenone. Toxins 2018;10:347. https://doi.org/ 10.3390/toxins 10090347

11. Robert H, Payros D, Pinton P, Théodorou V, Mercier-Bonin $\mathrm{M}$, Oswald IP. Impact of mycotoxins on the intestine: are mucus and microbiota new targets? J Toxicol Environ Health B 2017;20:249-75. https://doi.org/10.1080/10937404.2017. 1326071

12.Przybylska-Gornowicz B, Tarasiuk M, Lewczuk B, et al. The effects of low doses of two Fusarium toxins, zearalenone and deoxynivalenol, on the pig jejunum. A light and electron microscopic study. Toxins 2015;7:4684-705. https://doi.org/ 10.3390/toxins7114684

13.Liew WPP, Mohd-Redzwan S. Mycotoxin: its impact on gut health and microbiota. Front Cell Infect Microbiol 2018;8:60. https://doi.org/10.3389/fcimb.2018.00060

14.Committee on Nutrient Requirements of Swine, National Research Council. Nutrition requirements of swine. 11th ed. Washington, DC, USA: National Academies Press; 2012. https://doi.org/10.17226/13298

15. Reddy KE, Jeong JY, Lee Y, et al. Deoxynivalenol- and zearalenone-contaminated feeds alter gene expression profiles in the livers of piglets. Asian-Australas J Anim Sci 2018;31;595606. https://doi.org/10.5713/ajas.17.0466

16. Yu Z, Morrison M. Improved extraction of PCR-quality community DNA from digesta and fecal samples. Biotechniques 2004;36:808-12. https://doi.org/10.2144/04365ST04

17. Magoc T, Salzberg S. FLASH: fast length adjustment of short reads to improve genome assemblies. Bioinformatics 2011;27: 2957-63. https://doi.org/10.1093/bioinformatics/btr507

18. Caporaso JG, Kuczynski J, Stombaugh J, et al. QIIME allows analysis of high-throughput community sequencing data. Nat Methods 2010;7:335-6. https://doi.org/10.1038/nmeth. f.303

19.DeSantis TZ, Hugenholtz P, Larsen N, et al. Greengenes, a chimera-checked 16S rRNA gene database and workbench compatible with ARB. Appl Environ Microbiol 2006;72:506972. https://doi.org/10.1128/AEM.03006-05

20.Edgar RC. Search and clustering orders of magnitude faster than BLAST. Bioinformatics 2010;26:2460-1. https://doi.org/ 10.1093/bioinformatics/btq461

21.Hu P, Niu Q, Zhu Y, Shi C, Wang J, Zhu W. Effects of early commercial milk supplement on the mucosal morphology, bacterial community and bacterial metabolites in jejunum of the pre- and post-weaning piglets. Asian-Australas J Anim Sci 2020;33:480-9. https://doi.org/10.5713/ajas.18.0941

22.Piotrowska M, Slizewska K, Nowak A, et al. The effect of experimental fusarium mycotoxicosis on microbiota diversity in porcine ascending colon contents. Toxins 2014;6:206481. https://doi.org/10.3390/toxins6072064

23.Li F, Wang J, Huang LB, Chen H, Wang C. Effects of adding Clostridium sp. WJ06 on intestinal morphology and microbial diversity of growing pigs fed with natural deoxynivalenol contaminated wheat. Toxins 2017;9:383. https://doi.org/10. 3390/toxins 9120383

24. Isaacson R, Kim HB. The intestinal microbiome of the pig. Anim Health Res Rev 2012;13:100-9. https://doi.org/10. 1017/S1466252312000084

25. Mateos I, Combes S, Pascal G, et al. Fumonisin-exposure impairs age-related ecological succession of bacterial species in weaned pig gut microbiota. Toxins 2018;10:230. https:// doi.org/10.3390/toxins10060230

26. Pajarillo EAB, Chae JP, Balolong MP, Kim HB, Kang DK. Assessment of fecal bacterial diversity among healthy piglets during the weaning transition. J Gen Appl Microbiol 2014;60: 140-6. https://doi.org/10.2323/jgam.60.140 
27.Leser TD, Amenuvor JZ, Jensen TK, Lindecrona RH, Boye M, Moller K. Culture-independent analysis of gut bacteria: the pig gastrointestinal tract microbiota revisited. Appl Environ Microbiol 2002;68:673-90. https://doi.org/10.1128/AEM.68. 2.673-690.2002

28. Niu Q, Li P, Hao S, et al. Dynamic distribution of the gut microbiota and the relationship with apparent crude fiber digestibility and growth stages in pigs. Sci Rep 2015;5:9938. https://doi.org/10.1038/srep09938

29. Goyal N, Rishi P, Shukla G. Lactobacillus rhamnosus GG antagonizes Giardia intestinalis induced oxidative stress and intestinal disaccharidases: an experimental study. World J Microbiol Biotechnol 2013;29:1049-57. https://doi.org/10. 1007/s11274-013-1268-6

30. Yang J, Qian K, Wang C, Wu Y. Roles of probiotic lactobacilli inclusion in helping piglets establish healthy intestinal interenvironment for pathogen defense. Probiotics Antimicrob Proteins 2018;10:243-50. https://doi.org/10.1007/s12602017-9273-y

31. Walter J. Ecological role of lactobacilli in the gastrointestinal tract: implications for fundamental and biomedical research. Appl Environ Microbiol 2008;74:4985-96. https://doi.org/10. 1128/AEM.00753-08

32. Ertekin I, Kizilsimsek M. Effects of lactic acid bacteria inoculation in pre-harvesting period on fermentation and feed quality properties of alfalfa silage. Asian-Australas J Anim Sci 2020;33:245-53. https://doi.org/10.5713/ajas.18.0801

33.Jia T, Sun Z, Gao R, Yu Z. Lactic acid bacterial inoculant effects on the vitamin content of alfalfa and Chinese leymus silage. Asian-Australas J Anim Sci 2019;32:1873-81. https:// doi.org/10.5713/ajas.19.0135

34. Valeriano VD, Parungao-Balolong MM, Kang DK. In vitro evaluation of the mucin-adhesion ability and probiotic potential of Lactobacillus mucosae LM1. J Appl Microbiol 2014; 117:485-97. https://doi.org/10.1111/jam.12539

35. Giang HH, Viet TQ, Ogle B, Lindberg JE. Growth performance, digestibility, gut environment and health status in weaned piglets fed a diet supplemented with potentially probiotic complexes of lactic acid bacteria. Livest Sci 2010;129: 95-103. https://doi.org/10.1016/j.livsci.2010.01.010

36. Marin DE, T,ăranu I, Grosu H. Microorganisms involved in the decontamination of trichotecens, mycotoxins produced by Fusarium fungi. Arch Zootech 2011;14:5-24.

37. Franco TS, Garcia S, Hirooka EY, Ono YS, dos Santos JS. Lactic acid bacteria in the inhibition of Fusarium graminearum and deoxynivalenol detoxification. J Appl Microbiol 2011;111: 739-48. https://doi.org/10.1111/j.1365-2672.2011.05074.x

38. Yang WC, Hsu TC, Cheng KC, Liu JR. Expression of the Clonostachys rosea lactonohydrolase gene by Lactobacillus reuteri to increase its zearalenone-removing ability. Microb Cell Fact 2017;16:69. https://doi.org/10.1186/s12934-0170687-8

39. El-Nezami H, Polychronaki N, Salminen S, Mykkänen H. Binding rather than metabolism may explain the interaction of two food-grade Lactobacillus strains with zearalenone and its derivative a-zearalenol. Appl Environ Microbiol 2002;68:3545-9. https://doi.org/10.1128/AEM.68.7.35453549.2002

40.El-Nezami H, Mykkänen H, Kankaanpää P, Salminen S, Ahokas J. Ability of Lactobacillus and Propionibacterium strains to remove aflatoxin B1 from the chicken duodenum. J Food Prot 2000;63:549-52. https://doi.org/10.4315/0362028X-63.4.549

41.Tan H, Zhai Q, Chen W. Investigations of Bacteroides spp. towards next-generation probiotics. Food Res Int 2019;116: 637-44. https://doi.org/10.1016/j.foodres.2018.08.088

42. Saint-Cyr MJ, Perrin-Guyomard A, Houee P, Rolland JG, Laurentie M. Evaluation of an oral subchronic exposure of deoxynivalenol on the composition of human gut microbiota in a model of human microbiota-associated rats. PLoS One 2013;8:e80578. https://doi.org/10.1371/journal.pone.008 0578

43. Correa-Matos NJ, Donovan SM, Isaacson RE, Gaskins HR, White BA, Tappenden KA. Fermentable fiber reduces recovery time and improves intestinal function in piglets following Salmonella typhimurium infection. J Nutr 2003;133:184552. https://doi.org/10.1093/jn/133.6.1845

44. Kajihara Y, Yoshikawa S, Cho Y, Ito T, Miyamoto H, Kodama H. Preferential isolation of Megasphaera elsdenii from pig feces. Anaerobe 2017;48:160-4. https://doi.org/10.1016/j. anaerobe.2017.08.013

45.Liu Y. Fatty acids, inflammation and intestinal health in pigs. J Anim Sci Biotechnol 2015;6:41. https://doi.org/10.1186/ s40104-015-0040-1

46.Zilbauer M, Dorrell N, Wren BW, Bajaj-Elliott M. Campylobacter jejuni-mediated disease pathogenesis: an update. Trans R Soc Trop Med Hyg 2008;102:123-9. https:/doi.org/10.1016/ j.trstmh.2007.09.019

47. Burrough ER, Arruda BL, Patience JF, Plummer PJ. Alterations in the colonic microbiota of pigs associated with feeding distillers dried grains with solubles. PLoS One 2015;10: e0141337. https://doi.org/10.1371/journal.pone.0141337

48. Gratz SW, Currie V, Richardson AJ, et al. Porcine small and large intestinal microbiota rapidly hydrolyze the masked mycotoxin deoxynivalenol-3-glucoside and release deoxynivalenol in spiked batch cultures in vitro. Appl Environ Microbiol 2018;84:e02106-17. https://doi.org/10.1128/AEM. 02106-17 